\title{
REMARKS ON AN OPERATOR WIELANDT INEQUALITY*
}

\author{
PINGPING ZHANG ${ }^{\dagger}$
}

\begin{abstract}
Let $A$ be a positive operator on a Hilbert space $\mathcal{H}$ with $0<m \leq A \leq M$, and let $X$ and $Y$ be isometries on $\mathcal{H}$ such that $X^{*} Y=0, p>0$, and $\Phi$ be a 2-positive unital linear map. Define

$$
\Gamma=\left(\Phi\left(X^{*} A Y\right) \Phi\left(Y^{*} A Y\right)^{-1} \Phi\left(Y^{*} A X\right)\right)^{p} \Phi\left(X^{*} A X\right)^{-p} .
$$

Several upper bounds for $\frac{1}{2}\left|\Gamma+\Gamma^{*}\right|$ are established. These bounds complement a recent result on operator Wielandt inequality.
\end{abstract}

Key words. Operator inequality, Wielandt inequality, 2-Positive linear map, Partial isometry.

AMS subject classifications. 47A63, 47A30.

1. Introduction. Throughout we reserve $M$ and $m$ for scalars, and I for the identity operator. Other capital letters denote general elements of the $C^{*}$-algebra $\mathcal{B}(\mathcal{H})$ (with unit) of all bounded linear operator acting on a Hilbert space $(\mathcal{H},\langle\cdot, \cdot\rangle)$. Also, we identify a scalar with the unit multiplied by this scalar. The operator norm is denoted by $\|\cdot\|$. In this article, the inequality between operators is in the sense of Loewner partial order, that is, $T \geq S$ (the same as $S \leq T$ ) means that $T-S$ is positive. A positive invertible operator $T$ is naturally denoted by $T>0$.

A linear map $\Phi$ is positive if $\Phi(A) \geq 0$ whenever $A \geq 0$. It is said to be unital if $\Phi(\mathrm{I})=\mathrm{I}$. We say that $\Phi$ is 2 -positive if whenever the $2 \times 2$ operator matrix $\left[\begin{array}{cc}A & B \\ B^{*} & C\end{array}\right]$ is positive, then so is $\left[\begin{array}{cc}\Phi(A) & \Phi(B) \\ \Phi\left(B^{*}\right) & \Phi(C)\end{array}\right]$.

The Wielandt inequality [3, p. 443] states that if $0<m \leq A \leq M$, and $x, y \in \mathcal{H}$

*Received by the editors on May 22, 2014. Accepted for publication on September 22, 2015. Handling Editor: Bryan L. Shader.

${ }^{\dagger}$ School of Science, Chongqing University of Posts and Telecommunications, Chongqing, 400065, People's Republic of China (zhpp04010248@126.com). This work was supported by the Foundation of Chongqing University of Posts and Telecommunications for the Scholars with Doctorate (no. A2014-16), the Youth Science Research Project of Chongqing University of Posts and Telecommunications (no. A2014-104), and the Basic and Advanced Research Project of CQ CSTC (no. cstc2014jcyjA00044). 
with $x \perp y$, then

$$
|\langle x, A y\rangle|^{2} \leq\left(\frac{M-m}{M+m}\right)^{2}\langle x, A y\rangle\langle y, A x\rangle .
$$

Bhatia and Davis [1, Theorem 2] proved that if $0<m \leq A \leq M$ and $X$ and $Y$ are partial isometries on $\mathcal{H}$ whose final spaces are orthogonal to each other, then for every 2 -positive linear map $\Phi$

$$
\Phi\left(X^{*} A Y\right) \Phi\left(Y^{*} A Y\right)^{-1} \Phi\left(Y^{*} A X\right) \leq\left(\frac{M-m}{M+m}\right)^{2} \Phi\left(X^{*} A X\right) .
$$

Thus, inequality (1.1) can be viewed as an operator version of Wielandt inequality. In 2013, Lin presented the following conjecture.

Conjecture. [5, Conjecture 3.4] Let $A$ be a positive operator on a Hilbert space $\mathcal{H}$ with $0<m \leq A \leq M$, and let $X$ and $Y$ be partial isometries on $\mathcal{H}$ whose final spaces are orthogonal to each other. Then for every 2-positive linear map $\Phi$

$$
\left\|\Phi\left(X^{*} A Y\right) \Phi\left(Y^{*} A Y\right)^{-1} \Phi\left(Y^{*} A X\right) \Phi\left(X^{*} A X\right)^{-1}\right\| \leq\left(\frac{M-m}{M+m}\right)^{2} .
$$

Under the assumption that the conjecture is valid, the following two inequalities follow without much additional effort

$$
\frac{1}{2}\left|\Gamma+\Gamma^{*}\right| \leq\left(\frac{M-m}{M+m}\right)^{2}
$$

and

$$
\frac{1}{2}\left(\Gamma+\Gamma^{*}\right) \leq\left(\frac{M-m}{M+m}\right)^{2}
$$

where $\Gamma=\Phi\left(X^{*} A Y\right) \Phi\left(Y^{*} A Y\right)^{-1} \Phi\left(Y^{*} A X\right) \Phi\left(X^{*} A X\right)^{-1}$.

If one can drop the strong assumption on the validity of the conjecture which is still open, then apparently (1.2) and (1.3) are very attractive alternative operator versions of Wielandt inequality. Thus, it is interesting to estimate $\frac{1}{2}\left|\Gamma+\Gamma^{*}\right|$ and $\frac{1}{2}\left(\Gamma+\Gamma^{*}\right)$ without the assumption that the conjecture is true. This is our main motivation.

In this article, we consider a more general $\Gamma$ defined as

$$
\Gamma=\left(\Phi\left(X^{*} A Y\right) \Phi\left(Y^{*} A Y\right)^{-1} \Phi\left(Y^{*} A X\right)\right)^{p} \Phi\left(X^{*} A X\right)^{-p}, \quad p>0,
$$

where $X, Y$ are isometries such that $X^{*} Y=0$, and $\Phi$ is a 2-positive unital linear map. Three upper bounds for both $\frac{1}{2}\left|\Gamma+\Gamma^{*}\right|$ and $\frac{1}{2}\left(\Gamma+\Gamma^{*}\right)$ are presented. 
2. Main results. The following two lemmas are needed in our main results.

Lemma 2.1. [4, Lemma 3.5.12] For any bounded operator $X$,

$$
|X| \leq t \mathrm{I} \quad \Leftrightarrow \quad\|X\| \leq t \quad \Leftrightarrow \quad\left[\begin{array}{cc}
t \mathrm{I} & X \\
X^{*} & t \mathrm{I}
\end{array}\right] \geq 0 .
$$

Lemma 2.2. [2, Theorem 6] Let $0 \leq A \leq B$ and $0<m \leq A \leq M$. Then

$$
A^{2} \leq \frac{(M+m)^{2}}{4 M m} B^{2}
$$

REMARK 1. The condition $0<m \leq A \leq M$ in Lemma 2.2 can be replaced by $0<m \leq B \leq M$ by applying the continuity of operator and operator reverse monotonicity of the inverse.

Now we present the main results of this paper.

ThEOREM 2.3. Let $A$ be a positive operator on a Hilbert space $\mathcal{H}$ with $0<m \leq$ $A \leq M$, and let $X$ and $Y$ be isometries on $\mathcal{H}$ such that $X^{*} Y=0, p>0$, and $\Phi$ be a 2-positive unital linear map. Define

$$
\Gamma=\left(\Phi\left(X^{*} A Y\right) \Phi\left(Y^{*} A Y\right)^{-1} \Phi\left(Y^{*} A X\right)\right)^{p} \Phi\left(X^{*} A X\right)^{-p} .
$$

Then

$$
\frac{1}{2}\left|\Gamma+\Gamma^{*}\right| \leq \frac{\left(\frac{M-m}{M+m}\right)^{4 p} M^{2 p}+m^{-2 p}}{2}
$$

and

$$
\frac{1}{2}\left(\Gamma+\Gamma^{*}\right) \leq \frac{\left(\frac{M-m}{M+m}\right)^{4 p} M^{2 p}+m^{-2 p}}{2}
$$

Proof. We need the following simple fact:

$$
\|A B+B A\| \leq\left\|A^{2}+B^{2}\right\|, \quad \text { if } A \geq 0 \text { and } B \geq 0 .
$$

Thus, (1.1) and (2.3) imply

$$
\begin{aligned}
\frac{1}{2}\left\|\Gamma+\Gamma^{*}\right\| & \leq \frac{1}{2}\left\|\left(\Phi\left(X^{*} A Y\right) \Phi\left(Y^{*} A Y\right)^{-1} \Phi\left(Y^{*} A X\right)\right)^{2 p}+\Phi\left(X^{*} A X\right)^{-2 p}\right\| \\
& \leq \frac{1}{2}\left(\left\|\Phi\left(X^{*} A Y\right) \Phi\left(Y^{*} A Y\right)^{-1} \Phi\left(Y^{*} A X\right)\right\|^{2 p}+\left\|\Phi\left(X^{*} A X\right)^{-1}\right\|^{2 p}\right)
\end{aligned}
$$




$$
\begin{aligned}
& \leq \frac{1}{2}\left(\left\|\left(\frac{M-m}{M+m}\right)^{2} \Phi\left(X^{*} A X\right)\right\|^{2 p}+\left\|\Phi\left(X^{*} A X\right)^{-1}\right\|^{2 p}\right) \\
& \leq \frac{\left(\frac{M-m}{M+m}\right)^{4 p} M^{2 p}+m^{-2 p}}{2} .
\end{aligned}
$$

The last inequality above holds since $X^{*} X=\mathrm{I}$ and $0<m \leq A \leq M$, then $m \leq$ $X^{*} A X \leq M$ and so $m \leq \Phi\left(X^{*} A X\right) \leq M, \frac{1}{M} \leq \Phi\left(X^{*} A X\right)^{-1} \leq \frac{1}{m}$.

Inequality (2.1) follows from Lemma 2.1 and inequality (2.4). Inequality (2.2) holds from (2.1) and the fact that $|X| \geq X$ for any self-adjoint $X$. Thus, we complete the proof. $\square$

A sharper result is the following:

TheOREm 2.4. Under the same condition as in Theorem 2.3. Then

$$
\frac{1}{2}\left|\Gamma+\Gamma^{*}\right| \leq \begin{cases}\left(\frac{M-m}{M+m}\right)^{2 p}, & \text { when } 0<p \leq \frac{1}{2} \\ \left(\frac{M-m}{M+m}\right)^{2 p}\left(\frac{M}{m}\right)^{p}, & \text { when } p>\frac{1}{2}\end{cases}
$$

Consequently,

$$
\frac{1}{2}\left(\Gamma+\Gamma^{*}\right) \leq \begin{cases}\left(\frac{M-m}{M+m}\right)^{2 p}, & \text { when } 0<p \leq \frac{1}{2} \\ \left(\frac{M-m}{M+m}\right)^{2 p}\left(\frac{M}{m}\right)^{p}, & \text { when } p>\frac{1}{2}\end{cases}
$$

Proof. Combining the inequality (1.1) and the fact that $f(t)=t^{r}$ is operator monotone for $0 \leq r \leq 1$, we have

$$
\left(\Phi\left(X^{*} A Y\right) \Phi\left(Y^{*} A Y\right)^{-1} \Phi\left(Y^{*} A X\right)\right)^{p} \leq\left(\frac{M-m}{M+m}\right)^{2 p} \Phi\left(X^{*} A X\right)^{p},
$$

when $0<p \leq 1$. Inequality (2.7) is equivalent to

$$
\|\Gamma\| \leq\left(\frac{M-m}{M+m}\right)^{2 p}
$$

when $0<p \leq \frac{1}{2}$.

By Lemma 2.1 and inequality (2.8), we have

$$
\left[\begin{array}{cc}
\left(\frac{M-m}{M+m}\right)^{2 p} \mathrm{I} & \Gamma \\
\Gamma^{*} & \left(\frac{M-m}{M+m}\right)^{2 p} \mathrm{I}
\end{array}\right] \geq 0 \quad \text { and } \quad\left[\begin{array}{cc}
\left(\frac{M-m}{M+m}\right)^{2 p} & \Gamma^{*} \\
\Gamma & \left(\frac{M-m}{M+m}\right)^{2 p} \mathrm{I}
\end{array}\right] \geq 0
$$


Summing up these two operator matrices, we have

$$
\left[\begin{array}{cc}
2\left(\frac{M-m}{M+m}\right)^{2 p} \mathrm{I} & \Gamma+\Gamma^{*} \\
\Gamma^{*}+\Gamma & 2\left(\frac{M-m}{M+m}\right)^{2 p} \mathrm{I}
\end{array}\right] \geq 0 .
$$

It follows from Lemma 2.1 again that

$$
\frac{1}{2}\left|\Gamma+\Gamma^{*}\right| \leq\left(\frac{M-m}{M+m}\right)^{2 p}
$$

when $0<p \leq \frac{1}{2}$. When $p>\frac{1}{2}$, it follows that

$$
\begin{aligned}
\|\Gamma\| & \leq\left\|\left(\Phi\left(X^{*} A Y\right) \Phi\left(Y^{*} A Y\right)^{-1} \Phi\left(Y^{*} A X\right)\right)^{p}\right\|\left\|\Phi\left(X^{*} A X\right)^{-p}\right\| \\
& =\left\|\Phi\left(X^{*} A Y\right) \Phi\left(Y^{*} A Y\right)^{-1} \Phi\left(Y^{*} A X\right)\right\|^{p}\left\|\Phi\left(X^{*} A X\right)^{-1}\right\|^{p} \\
& \leq\left\|\left(\frac{M-m}{M+m}\right)^{2} \Phi\left(X^{*} A X\right)\right\|^{p}\left\|\Phi\left(X^{*} A X\right)^{-1}\right\|^{p} \quad \text { (by the inequality (1.1)) } \\
(2.10) & \leq\left(\frac{M-m}{M+m}\right)^{2 p}\left(\frac{M}{m}\right)^{p} .
\end{aligned}
$$

Similarly,

$$
\frac{1}{2}\left|\Gamma+\Gamma^{*}\right| \leq\left(\frac{M-m}{M+m}\right)^{2 p}\left(\frac{M}{m}\right)^{p}
$$

when $p>\frac{1}{2}$. Inequality (2.5) holds by inequalities (2.9) and (2.11). While inequality (2.6) holds by inequality (2.5). This completes the proof of Theorem 2.4, $\square$

REMARK 2. We provide a second method to prove the inequality (2.5):

$$
\left\|\frac{\Gamma+\Gamma^{*}}{2}\right\| \leq\|\Gamma\|
$$

which together with (2.8), (2.10) and Lemma 2.1 leads to the inequality (2.5).

REMARK 3. The bounds in Theorem 2.4 are tighter than those in Theorem 2.3 by the following inequalities

$$
\frac{\left(\frac{M-m}{M+m}\right)^{4 p} M^{2 p}+m^{-2 p}}{2} \geq\left(\frac{M-m}{M+m}\right)^{2 p}\left(\frac{M}{m}\right)^{p} \geq\left(\frac{M-m}{M+m}\right)^{2 p}
$$

The first inequality above holds by the scalar AM-GM inequality, and the second inequality holds since $m \leq M$ and $p>0$. 
Our last result is the following:

ThEOREM 2.5. Under the same condition as in Theorem 2.3, Then

$$
\frac{1}{2}\left|\Gamma+\Gamma^{*}\right| \leq\left(\frac{M-m}{M+m}\right)^{2 p}\left(\frac{\left(\frac{M}{m}\right)^{\frac{p}{2}}+\left(\frac{m}{M}\right)^{\frac{p}{2}}}{2}\right)^{\lceil p\rceil}
$$

where $\lceil p\rceil$ is the ceiling function of $p$. Consequently,

$$
\frac{1}{2}\left(\Gamma+\Gamma^{*}\right) \leq\left(\frac{M-m}{M+m}\right)^{2 p}\left(\frac{\left(\frac{M}{m}\right)^{\frac{p}{2}}+\left(\frac{m}{M}\right)^{\frac{p}{2}}}{2}\right)^{\lceil p\rceil}
$$

Proof. Firstly, we prove the following inequality

$$
\|\Gamma\| \leq\left(\frac{M-m}{M+m}\right)^{2 p}\left(\frac{\left(\frac{M}{m}\right)^{\frac{p}{2}}+\left(\frac{m}{M}\right)^{\frac{p}{2}}}{2}\right)^{\lceil p\rceil}
$$

The proof of (2.14) is proved by induction on $\lceil p\rceil$. For $\lceil p\rceil=1$, i.e., $0<p \leq 1$, combining (2.7) and Lemma 2.2 gives

$$
\begin{aligned}
\left(\Phi\left(X^{*} A Y\right) \Phi\left(Y^{*} A Y\right)^{-1} \Phi\left(Y^{*} A X\right)\right)^{2 p} \\
\quad \leq\left(\frac{M-m}{M+m}\right)^{4 p} \frac{\left(M^{p}+m^{p}\right)^{2}}{4 M^{p} m^{p}} \Phi\left(X^{*} A X\right)^{2 p} \\
\quad=\left(\frac{M-m}{M+m}\right)^{4 p}\left(\frac{\left(\frac{M}{m}\right)^{\frac{p}{2}}+\left(\frac{m}{M}\right)^{\frac{p}{2}}}{2}\right)^{2} \Phi\left(X^{*} A X\right)^{2 p}
\end{aligned}
$$

which is equivalent to

$$
\|\Gamma\| \leq\left(\frac{M-m}{M+m}\right)^{2 p} \frac{\left(\frac{M}{m}\right)^{\frac{p}{2}}+\left(\frac{m}{M}\right)^{\frac{p}{2}}}{2}=\left(\frac{M-m}{M+m}\right)^{2 p}\left(\frac{\left(\frac{M}{m}\right)^{\frac{p}{2}}+\left(\frac{m}{M}\right)^{\frac{p}{2}}}{2}\right)^{\lceil p\rceil} .
$$

Thus, inequality (2.14) holds for $\lceil p\rceil=1$.

Now, suppose that inequality (2.14) holds for $\lceil p\rceil \leq k(k \geq 1)$. That is

$$
\|\Gamma\| \leq\left(\frac{M-m}{M+m}\right)^{2 p}\left(\frac{\left(\frac{M}{m}\right)^{\frac{p}{2}}+\left(\frac{m}{M}\right)^{\frac{p}{2}}}{2}\right)^{\lceil p\rceil}
$$

which is equivalent to 


$$
\begin{aligned}
& \left(\Phi\left(X^{*} A Y\right) \Phi\left(Y^{*} A Y\right)^{-1} \Phi\left(Y^{*} A X\right)\right)^{2 p} \\
& \leq\left(\frac{M-m}{M+m}\right)^{4 p}\left(\frac{\left(\frac{M}{m}\right)^{\frac{p}{2}}+\left(\frac{m}{M}\right)^{\frac{p}{2}}}{2}\right)^{2\lceil p\rceil} \Phi\left(X^{*} A X\right)^{2 p} .
\end{aligned}
$$

Combining inequality (2.15) and Lemma 2.2 leads to

$$
\begin{aligned}
(\Phi & \left.\left(X^{*} A Y\right) \Phi\left(Y^{*} A Y\right)^{-1} \Phi\left(Y^{*} A X\right)\right)^{4 p} \\
& \leq\left(\frac{M-m}{M+m}\right)^{8 p}\left(\frac{\left(\frac{M}{m}\right)^{\frac{p}{2}}+\left(\frac{m}{M}\right)^{\frac{p}{2}}}{2}\right)^{4\lceil p\rceil} \frac{\left(M^{2 p}+m^{2 p}\right)^{2}}{4 M^{2 p} m^{2 p}} \Phi\left(X^{*} A X\right)^{4 p} \\
& =\left(\frac{M-m}{M+m}\right)^{8 p}\left(\frac{\left(\frac{M}{m}\right)^{\frac{p}{2}}+\left(\frac{m}{M}\right)^{\frac{p}{2}}}{2}\right)^{4\lceil p\rceil}\left(\frac{\left(\frac{M}{m}\right)^{p}+\left(\frac{m}{M}\right)^{p}}{2}\right)^{2} \Phi\left(X^{*} A X\right)^{4 p} \\
& \leq\left(\frac{M-m}{M+m}\right)^{8 p}\left(\frac{\left(\frac{M}{m}\right)^{p}+\left(\frac{m}{M}\right)^{p}}{2}\right)^{2\lceil p\rceil}\left(\frac{\left(\frac{M}{m}\right)^{p}+\left(\frac{m}{M}\right)^{p}}{2}\right)^{2} \Phi\left(X^{*} A X\right)^{4 p} \\
& =\left(\frac{M-m}{M+m}\right)^{8 p}\left(\frac{\left(\frac{M}{m}\right)^{p}+\left(\frac{m}{M}\right)^{p}}{2}\right)^{2(\lceil p\rceil+1)} \Phi\left(X^{*} A X\right)^{4 p} \\
(2.16) & \leq\left(\frac{M-m}{M+m}\right)^{8 p}\left(\frac{\left(\frac{M}{m}\right)^{p}+\left(\frac{m}{M}\right)^{p}}{2}\right)^{2(k+1)} \Phi\left(X^{*} A X\right)^{4 p},
\end{aligned}
$$

where $\lceil p\rceil \leq k$. Inequality (2.16) is equivalent to

$$
\begin{aligned}
& \left(\Phi\left(X^{*} A Y\right) \Phi\left(Y^{*} A Y\right)^{-1} \Phi\left(Y^{*} A X\right)\right)^{2 p} \\
& \leq\left(\frac{M-m}{M+m}\right)^{4 p}\left(\frac{\left(\frac{M}{m}\right)^{\frac{p}{2}}+\left(\frac{m}{M}\right)^{\frac{p}{2}}}{2}\right)^{2(k+1)} \Phi\left(X^{*} A X\right)^{2 p},
\end{aligned}
$$

where $\lceil p\rceil \leq 2 k$. Since $k+1 \leq 2 k$ when $k \geq 1$, inequality (2.17) holds for $\lceil p\rceil=k+1$, i.e.,

$$
\begin{aligned}
\left(\Phi\left(X^{*} A Y\right) \Phi\left(Y^{*} A Y\right)^{-1} \Phi\left(Y^{*} A X\right)\right)^{2 p} \\
\leq\left(\frac{M-m}{M+m}\right)^{4 p}\left(\frac{\left(\frac{M}{m}\right)^{\frac{p}{2}}+\left(\frac{m}{M}\right)^{\frac{p}{2}}}{2}\right)^{2(k+1)} \Phi\left(X^{*} A X\right)^{2 p} \\
=\left(\frac{M-m}{M+m}\right)^{4 p}\left(\frac{\left(\frac{M}{m}\right)^{\frac{p}{2}}+\left(\frac{m}{M}\right)^{\frac{p}{2}}}{2}\right)^{2\lceil p\rceil} \Phi\left(X^{*} A X\right)^{2 p}
\end{aligned}
$$

which is equivalent to

$$
\|\Gamma\| \leq\left(\frac{M-m}{M+m}\right)^{2 p}\left(\frac{\left(\frac{M}{m}\right)^{\frac{p}{2}}+\left(\frac{m}{M}\right)^{\frac{p}{2}}}{2}\right)^{\lceil p\rceil}
$$


when $\lceil p\rceil=k+1$. Thus, inequality (2.14) holds for $\lceil p\rceil=k+1$. This completes the induction.

Combining inequality (2.14), Remark 2 and Lemma 2.1) leads to inequality (2.12). Similarly, inequality (2.13) holds by inequality (2.12).

REMARK 4. Since $\frac{\left(\frac{M}{m}\right)^{\frac{p}{2}}+\left(\frac{m}{M}\right)^{\frac{p}{2}}}{2} \geq 1$, the bounds in Theorem 2.4 are sharper than those in Theorem 2.5 when $0<p \leq \frac{1}{2}$. It follows from the fact $\frac{m}{M} \leq 1 \leq \frac{M}{m}$ that $\frac{\left(\frac{M}{m}\right)^{\frac{p}{2}}+\left(\frac{m}{M}\right)^{\frac{p}{2}}}{2} \leq\left(\frac{M}{m}\right)^{\frac{p}{2}}$. Thus, the bounds in Theorem 2.5 are tighter than those in Theorem 2.4 when $\frac{1}{2}<p \leq 2$. When $p$ is large enough, such as $p>2+2 \log _{\frac{M}{m}} 2$, the bounds in Theorem 2.4 are tighter than those in Theorem 2.5 by the following inequalities

$$
\left(\frac{M}{m}\right)^{\frac{p}{2}}>2 \frac{M}{m}, \quad \text { if } \quad p>2+2 \log _{\frac{M}{m}} 2
$$

and

$$
\left(\frac{\left(\frac{M}{m}\right)^{\frac{p}{2}}+\left(\frac{m}{M}\right)^{\frac{p}{2}}}{2}\right)^{\lceil p\rceil}>\left(\frac{\left(\frac{M}{m}\right)^{\frac{p}{2}}}{2}\right)^{\lceil p\rceil} \geq\left(\frac{\left(\frac{M}{m}\right)^{\frac{p}{2}}}{2}\right)^{p} .
$$

Therefore, neither of the bounds in Theorem 2.4 and Theorem[2.5]are uniformly better than the other.

Acknowledgment. The author thanks the referee for the valuable comments and suggestions which improve the presentation of the paper.

\section{REFERENCES}

[1] R. Bhatia and C. Davis. More operator versions of the Schwarz inequality. Comm. Math. Phys., 215(2):239-244, 2000.

[2] M. Fujii, S. Izumino, R. Nakamato, and Y. Seo. Operator inequalities related to CauchySchwarz and Hölder-McCarthy inequalities. Nihonkai Math. J., 8(2):117-122, 1997.

[3] R.A. Horn and C.R. Johnson. Matrix Analysis. Cambridge University Press, Cambridge, 1985.

[4] R.A. Horn and C.R. Johnson. Topics in Matrix Analysis. Cambridge University Press, Cambridge, 1991.

[5] M. Lin. On an operator Kantorovich inequality for positive linear maps. J. Math. Anal. Appl., 402(1):127-132, 2013. 